estimated that up to $10^{-12} \mathrm{~W}$ of output power in a linewidth of a few millihertz is emitted from this novel laser, enough to be amplified and used in applications.

The natural linewidth of the atomic transition that is used to produce radiation in this set-up is much less than the linewidth of the cavity. In this sense, rather than to a common laser, the set-up better corresponds to a maser ${ }^{5}$ - the workhorse of frequency metrology where, for example, hydrogen atoms are made to emit narrow-linewidth microwave radiation at $1.4 \mathrm{GHz}$ into a microwave cavity. In the hydrogen maser, a continuous flux of excited atoms replenishes the system with energy lost to emission. In the proposal of Meiser and colleagues ${ }^{1}$, this energy is supplied by a rather slow excitation of the atoms by an additional laser field that pumps the trapped atoms back to the excited level of the laser transition. This approach has the benefit that length fluctuations of the cavity have only a minor effect on the frequency of the emitted radiation.

The proposal is a new approach to the next generation of ultrastable lasers. There are still some open questions: the set-up is experimentally quite demanding, as the atoms have to be held within a small volume defined by the cavity; technical noise might broaden the line; and the back-action of the photon recoil on the cavity and laser fields has so far not been taken into account. Hopefully, an experimental realization will soon show how well these issues can be handled in practice: the race is now on against cryogenic cavities, new materials and other optical designs.

Unquestionably, highly stable lasers will lead to high-stability clocks and enable the further exploitation of atomic reference lines, with a projected stability in lattice clocks of less than $10^{-16}$ within a one-second averaging time. The ability to compare different clocks leads to improved limits on the possible drift of fundamental constants in physics, and on tests of local position invariance. The effect of gravity on clocks will then become easily visible (a difference in height of only $1 \mathrm{~cm}$ leads to a general-relativistic time dilation of $10^{-18}$ ), making possible fascinating new applications for clocks.

Uwe Sterr ${ }^{\star}$ and Christian Lisdat are at the Physikalisch-Technische Bundesanstalt, Bundesallee 100, 38116 Braunschweig, Germany.

*e-mail: uwe.sterr@ptb.de

\section{References}

1. Meiser, D., Ye, J., Carlson, D. R. \& Holland, M. J. Phys. Rev. Lett. 102, 163601 (2009).

2. Hosaka, K. et al. Phys. Rev. A 79, 033403 (2009).

3. Young, B. C., Cruz, F. C., Itano, W. M. \& Bergquist, J. C. Phys. Rev. Lett. 82, 3799-3802 (1999).

4. Numata, K., Kemery, A. \& Camp, J. Phys. Rev. Lett. 93, 250602 (2004).

5. Goldenberg, H. M., Kleppner, D. \& Ramsey, N. F. Phys. Rev. Lett. 5, 361-365 (1960)

\title{
HISTORY OF QUANTUM THEORY
}

\section{The short version}

"Quantum mechanics is a difficult theory, the history of which is even more difficult." Such is not the conclusion, but the starting point of a study by Olivier Darrigol, in which he sets out to give a simplified account of the complex history of quantum mechanics, and its early history in particular (Studies in History and Philosophy of Modern Physics 40, 151-166; 2009). His "simplified genesis", Darrigol hopes, might serve both physicists and philosophers as a more direct approach to the foundations of quantum theory.

Darrigol considers the period from Max Planck's quantum hypothesis to the first complete mathematical formalism of quantum mechanics, Paul Dirac's transformation theory. During that time, spanning the first quarter of the last century, a number of great minds left their mark, as they investigated a broad spectrum of physical phenomena. Different schools emerged, in terms both of geographical location and of approach, and by the middle of the 1920s, two distinct formulations of quantum mechanics had emerged: matrix mechanics (developed in Germany by Werner Heisenberg, Max Born and Pascual Jordan, and by Dirac in England) and Erwin Schrödinger's wave mechanics.

Darrigol takes these two branches their formal equivalence was established

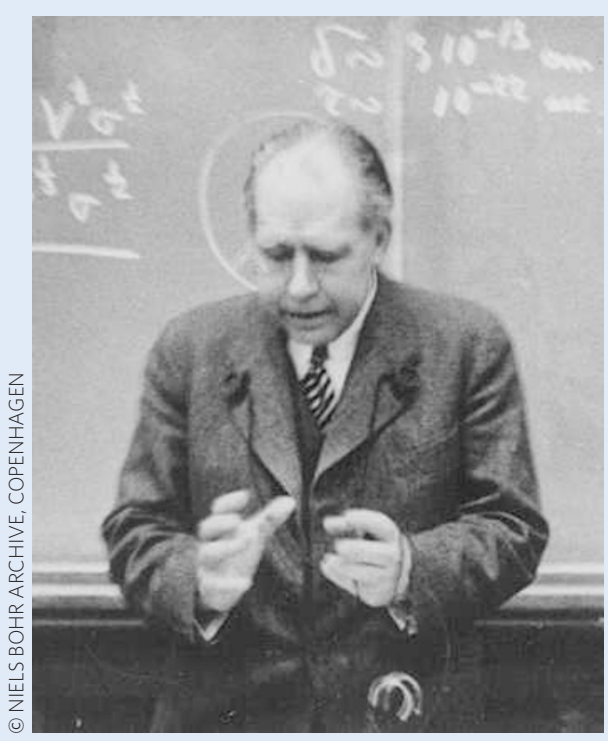

eventually - as the backbone of his 'simplified history'. He starts the story of matrix mechanics with the failure of classical electrodynamics to describe black-body radiation, leading to the work of Niels Bohr (pictured) on the model of atomic structure and the correspondence principle, and, in what Darrigol says may be regarded as a "necessary consequence" - Heisenberg's quantum mechanics. The developments that eventually led to wave mechanics, on the other hand, he traces back to Einstein's light-quantum hypothesis and its extension to matter waves, by Louis de Broglie.

Although this overall structure of Darrigol's 'brief history' might be, in itself, not surprising, it is in the selection of key contributions that he chooses to take a new path, so as to construct a coherent sequence of achievements where each step follows as a consequence of previous ones (anything but an easy task in the face of the multilayered history of the field). Also, convoluted derivations are replaced with shorter, more direct reasoning. This approach, Darrigol admits, does leave out important developments, and, in a sense, provides the kind of "linear, great-men accounts" which, in principle, should be avoided in historical writing. But in the light of the already existing large body of work covering the history of quantum mechanics, priority is given to a short and clear account that highlights conceptual connections and key features of quantum theory, in a way that facilitates capturing its foundations, as well as the philosophical stance of some of its fathers. A fuller history, Darrigol argues, would not alter much the basic constructive steps in his simplified genesis, or help to understand why quantum mechanics was born.

ANDREAS TRABESINGER 